\title{
Race As An Adverse Childhood Experience
}

Serena Bradshaw, Karen Oehme J.D, \& Ann Perko J.D Department of Social Work, Florida State University

\section{Introduction:}

According to the Center for Disease Control (CDC) adverse childhood experiences have both positive and negative effects on future violence victimization and on one's mental health.

Pustance Abuse and Mental Health services Administration, these ACEs include physical abuse, sexual abuse, emotional abuse, physical neglect,

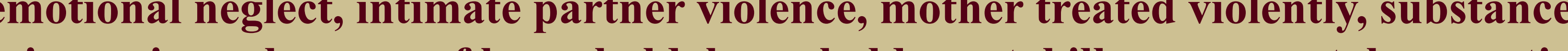

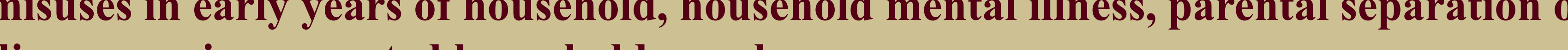
divorce, or incarcerated household members.

dear pipeline which includes: disrapted neurodevelopment, social, emotional, and cognitive behaviors; adoption Weath We conducted research on the (ACEs) scalte and aboul pacial tranna and concluded that race is an ad Acial trauma,

pecially in the black community can affect one's mental health and ffect their psyche. Research has concluded also that one does not have to erience racial discrimination, but the simple act of being black is a traumatic

We came to the provocative conclusion that though the black community was being Wecifically researched, all minority race groups experience racial trauma and that hinders them from healing or resiliency without the proper tools to treat this. Adding this sub factor of race as an (ACEs) could be used with resilience therapy that leads to healing efforts of marginalized communities in relation to trauma.

\section{Abstract:}

The purpose of this study was to explore the issue of race as a trauma related to The in the role of healing. The goal of this research is to help researchers think about the profound effects of race and help strengthen the ACEs scale. The ACEs scale is a scale that identifies trauma which is linked to later life well-being and health. Identifying ACEs, carefully understanding them, and providing preventative measures allow for healing and resitence buildig. Thi research is not he first of is kind, but is the first that include, but were not limited to: ACES, black trauma, post-enslavement, the psyche of African Americans, and other dynamics in the black community which combine to make the assumption that race is a traumatic experience. There was also research on the similarities and parallels of trauma within other cultures and ethnic groups. This resulted in the provocative conclusion that race is in fact an adverse should be accounted for in the new ACEs scales in order to help ensure the well-being and mortality of certain populations. This is essential in ACEs for minority which will lead to better preventive measures, as well as healing efforts.

\section{Methods:}
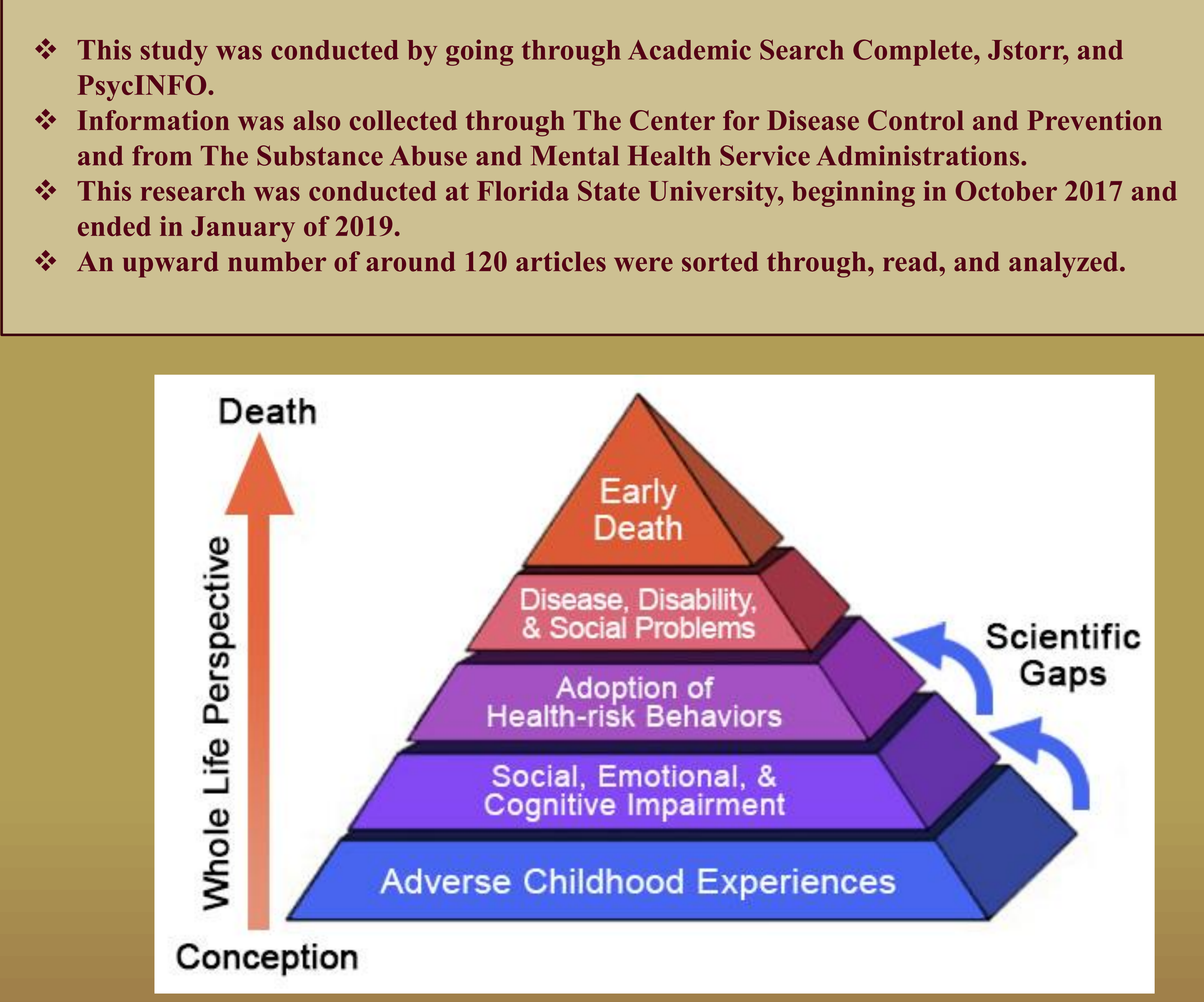

\section{Discussion:}

* Though every individual is different, the literature shows that these ACEs and trauma related issues are often caused by disparities that are linked to race and blackness which perpetrate a generational cycle of psychological warfare, inequality, and institutionalized and systematic racism.

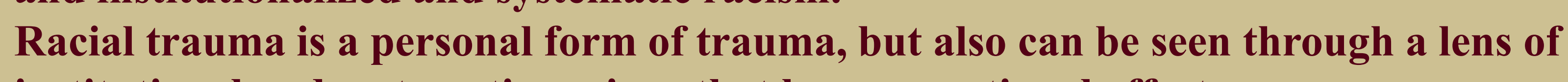
institutional and systematic racism, that has generational affects.

demographics were skewed. In terms of race and ethnicity participants, AfrionAmerican (Blacks) were the second to last group accounted for at $4.5 \%$, compared to Whites at $74.8 \%$, Hispanic / Latino populations at $11.2 \%$, Asian /Pacific Islander at $7.2 \%$ and the other category at $2.3 \%$ (Felitti et al., 1998).

All of the experiences seen in ACEs can be seen stackng upon each other and this creates issues that are no longer considered individual racism or prejudice but a systemic or institutionalized racism complex that is hard to debunk without proper

black commol trauma is seen in many forms such as the large medical disparity in the lack community, underfunded public school systems, racial profiling, epigenetics,

\section{Future Implications:}

Race may be viewed as a traumatic childhood experience and more specifically being black is an additional trauma that should be accounted for when utilizing (ACEs) scale.

It is important to analyze the communities in which these ACEs are typically the most prevalent in. In order to provide equality to all adverse backgrounds and experience in this culturally and politically charged climate, one must not ignore the affects that race has upon an individual growing up.

- Adding race as an ACEs would allow for the leverage between equality and equity. Adding race as a category in ACEs would also allow for proper understanding from pepple who also care to know more about populations affected by these issues and can aid in an ell
understand.

* We came to the provocative conclusion that though the black community was being

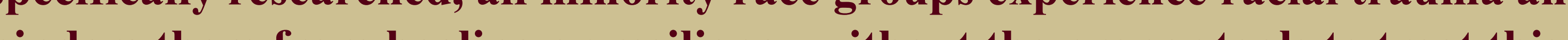

\section{References:}

(2016, June 14). Violence Prevention. Retrieved from https://www.cdc.gov/violenceprevention/acestudy/about htme

Collier-Thomas, B., \& Turner, J. (1994). Race, class and color: The African
American discourse on identity. Journal Of American Ethnic History, 14(1), 5 .

Felitti, V. J., Anda, R. F., Nordenberg, D., Williamson, D. F., Spitz, A. M., Edwards, V., ... Marks, J. S.

Relationship of childhood abuse and household dysfunction to many of the leading causes of death adults. The Adverse Childhood Experiences (ACE) Study. American Journal of Preventive Medicine, $14(4), 245-258$.

GRAFF, G. (2014). The interg
Psychohistory, 41(3), 181-197.

Shelton, J., Bryant, D., \& Brown, M. (2016). We, as a People?: Assessing the Consequences of Various Sources of Diversity within Black America. Phylon

Slater, A. (2014). W.E.B. Du Bois' Transationalism: Building a Collective Identity amoung the American Negro and the Asian Indian. Phylon (1960-), 51(1), 145-

Substance Abuse and Mental Health Services Administration. (2015, August 21). The Role of Adverse Childhood Experiences in Substance Abuse and Related Behavioral Health Problems. Retrieved from https://www.samhsa.gov/capt/prach
health/adverse-childhood-experiences

\section{Acknowledgements:}

I would like to express my gratitude to Dr.Oehme and Dr. Perko, for their continuous guidance and support throughout this project. Thank you. 